\title{
Upregulation of selected HERVW loci in multiple sclerosis
}

\author{
Sofía Macías-Redondo ${ }^{1}$, Mark Strunk ${ }^{2}$, Alberto Cebollada-Solanas ${ }^{3}$, José-Ramón Ara ${ }^{4,5}$, Jesús Martín ${ }^{4,5}$ and \\ Jon Schoorlemmer ${ }^{1,6,7^{*}}$
}

\section{Abstract and Introduction}

Human endogenous retrovirus (HERV) are the present day versions of retroviral germline infections that have occured millions of years ago, which occupy about $8 \%$ of the genome [1]. While they are mostly replication deficient, they are known to express RNA and protein [2] during particular developmental stages, or as a response to aging [3], inflammation and a wide range of pathologies [4]. A human retrovirus discovered in Multiple Sclerosis (MS) patients [5], turned out to be the prototype of a novel HERV family referred to as HERVW [6]. The HERV W family consists of 213 elements, 12 out of which are complete proviral copies with intact LTRs [7]. Increased expression of HERVW in peripheral blood mononuclear cells (PBMCs) has been repeatedly associated with MS, and the presence of HERVW protein or elevated RNA transcription has been correlated with disease activity [810]. While a contribution of HERVW-encoded proteins to brain disease is suggested by their presence in MSassociated brain lesions, expression in peripheral organs may be involved in the disease process through cytokineinduced damage to the blood brain barrier and subsequent infiltration of monocytes. Alterations in peripheral expression may also serve as a useful and practical marker for the diagnostics of this CNS disease. Therefore, we quantified overall HERVW levels and identified individual HERVW loci actually transcribed in PBMCs. Analysis was carried out in patients diagnosed with Clinically Isolated Syndrome (CIS), a precursor to MS, defined by a single episode of neurologic symptoms lasting at least $24 \mathrm{~h}$. CIS is an indicator of future development of MS, as $60 \%$ of

\footnotetext{
* Correspondence: jonas@araid.es

${ }^{1}$ Instituto Aragonés de Ciencias de la Salud (IACS), c/Juan Bosco 13, 50009

Zaragoza, Spain

${ }^{6}$ ARAID Foundation, Avda. de Ranillas 1-D, 50018 Zaragoza, Spain

Full list of author information is available at the end of the article
}

the people diagnosed with CIS develop MS [11]. These patients potentially represent the earliest stage of MS routinely available for clinical analysis. We undertook a Next Generation Sequencing (NGS)-based analysis of transcripts amplified from cDNA obtained from patients with CIS and samples from healthy controls. Data presented from this pilot experiment indicate that the relative frequency of specific HERVW copies is altered in PBMC of CIS patients, even in the absence of overall HERVW overexpression. Such altered frequency appears to be derived from less abundantly transcribed but potentially MSrelated HERVW loci.

\section{Methods \\ Patients (Table 1)}

The local ethics committee (CEICA) approved the study protocol (CP - CI PI14/0021 dated 26/02/2014; modified on 25/10/2017), and patients provided written informed consent (protocolo y información para el paciente v2 de 29/12/2013). Blood samples were collected from MS patients and healthy controls from the Neurology Department of Miguel Servet University Hospital (Zaragoza, Spain). Whole fresh blood was drawn into vacutainer tubes (Becton Dickinson Vacutainer) containing EDTA. Within $24 \mathrm{~h}$, PBMC were isolated as previously described $[12,13]$.

\section{Expression analysis and PCR}

RNA isolation and random-primed cDNA synthesis [14] was carried out as described before. HERVW ENV levels were determined by triplicate qPCR assays as described $[14,15]$. For the identification and localization of transcribed HERVW loci, cDNA was amplified employing the external primers of an established PCR assay for HERVW ENV [15]. Products were purified and subjected to NGS analysis. 
Table 1 Clinical features of MS patients included in this study

\begin{tabular}{|c|c|c|c|c|}
\hline Case MS subtype & Age & Sex & Status & RRMS designation \\
\hline RSM-49 & 25 & $\mathrm{~F}$ & NA & NO \\
\hline RSM-58 CIS & 44 & $\mathrm{~F}$ & A & YES \\
\hline RSM-70 CIS & 47 & $\mathrm{~F}$ & A & YES \\
\hline RSM-73 CIS & 39 & M & NA & YES \\
\hline RMS-103 CIS & 53 & $\mathrm{~F}$ & NA & YES \\
\hline RMS-114 CIS & 44 & M & NA & NO \\
\hline No MS Controls & Age & Sex & & \\
\hline RMS-3 & 36 & $\mathrm{~F}$ & & \\
\hline RMS-15 & 31 & M & & \\
\hline RMS-23 & 46 & $\mathrm{~F}$ & & \\
\hline RMS-30 & 46 & M & & \\
\hline RSM-42 & 39 & M & & \\
\hline RSM-46 & 44 & $M$ & & \\
\hline RSM-62 & 29 & $\mathrm{~F}$ & & \\
\hline RSM-72 & 46 & $M$ & & \\
\hline RSM-80 & 48 & $M$ & & \\
\hline RSM-98 & 47 & $M$ & & \\
\hline RSM-99* & 17 & M & & \\
\hline RSM-102* & 56 & $\mathrm{~F}$ & & \\
\hline RSM-104 & 44 & $M$ & & \\
\hline RMS-106* & 59 & $M$ & & \\
\hline RMS-109 & 45 & $M$ & & \\
\hline RMS-112 & 47 & $\mathrm{~F}$ & & \\
\hline RMS-113 & 28 & $M$ & & \\
\hline RMS-118 & 30 & $\mathrm{~F}$ & & \\
\hline
\end{tabular}

Clinical data of patients whose PBMCs were analyzed for HERVW expression. Median ages for both patients and controles groups were 44 years (mean and SEM are 42,0 +/- 4,25 and 40,4 +/- 1,94 for patient and control groups, respectively)

A/NA status refers to active and non-active patients respectively. Posterior progession towards MS diagnosis (RRMS) is indicated for all CIS cases. Samples analyzed by NGS are marked in blue. median ages in these groups are 44 year for patients and 47 years for controls. * indicates samples only analyzed by NGS

\section{NGS analysis}

Library preparation and sequencing was carried out using the IonTorrent technology workflow on an Ion Torrent S5XL platform using an Ion 530 chip. Resulting reads were mapped to the human reference genome (version hg19) using strict criteria to maximize mapping differences between different HERVW copies. Relative frequencies were calculated as the number of reads mapping to an individual HERVW ENV element relative to the total number of reads. Details in Suppl. Methods.

\section{Statistical analysis}

SPSS software was used for all analyses and graphs (Version 15.0). Normality and statistical significance of differences were assessed using specific tests. Data were further analyzed using the DESeq2 package [16] to correct $p$ values for multiple testing (False Discovery Rate $<0.05$ ).

More detailed information is available in Suppl. M\&M.

\section{Results}

We carried out HERVW ENV expression analyses using an optimized assay described by Mameli et al. [15]. No significantly increased expression of HERVW was detected in a small cohort of CIS patients $(n=6)$ compared to age-matched controls $(n=15)(\mathrm{U}-\mathrm{Mann}$-Whitney $p=$ 0.267) (Fig. 1). Results were not skewed by the use of $G A P D H$ as a reference gene (Fig. 1), as comparison with RPL19 and HSDA reference genes (Table S1 and Suppl Figure 1) showed that there is no statistical difference between the use of either GAPDH or the mean of the three genes (Welch's t-test; $p<0.05$ ).

In the absence of increased overall expression levels of HERVW in CIS samples, we wondered whether specific copies of HERVW (Table S2) might be differentially expressed. We performed NGS analysis to identify individual HERVW copies with altered expression in PBMC from CIS patients $(n=5)$ and controls $(n=5)$. Reads obtained $(70,694 \pm 24,812$ per sample; 25,286 $136,704)$ were mapped to the human genome. Once assigned to unique genomic locations, reads corresponding to 39 HERVW ENV loci were extracted (Table S3 and Table 2). As expected, $>99.85 \%$ of mapped reads correspond to the 39 loci analyzed (data not shown). The resulting data showed that reads obtained from CIS patients mapped to a significant higher number of different HERVW ENV loci $(31 \pm 13)$, compared to those obtained from controls $(16 \pm 5.5)$ (t-student; $p=$ 0.018 ) (Fig. 2 A). Over $70 \%$ of the reads mapped to either of two loci: 19q13.2, Xq22.3. Extending the range, reads mapped with high frequency $(>3.6 \%$ of total reads/locus) to a limited number of loci, in particular to HERVW ENV copies located on chromosomes 19q13.2, Xq22.3, 8q21.11, 15q21.3, 12q23.3 and 4q21.22 (Fig. 2 B). We found no significant differences between CIS patients and controls in the relative frequency of reads mapping to these loci (Fig. 2 B; Table 2).

Lower numbers of reads mapped to the remaining 33 loci, with relative frequencies ranging from 0,01 to $1,66 \%$. We found differences between CIS patients and controls in the relative frequency of reads mapping to several of these. Reads mapping to a subset of HERVW loci, including copy_chr3-1, copy_chr4-3, copy_chr5-1, copy_chr9-1, copy_chr9-2, copy_chr12-4 and copy_chr19-1 differed between CIS patients and controls (Table 3; Fig. 2 C-E). The relative frequency (ranging from $0,3-2,95 \%$ ) of reads mapping to these loci showed $a>7$ fold increase in CIS patients compared to controls (Table 3). When corrected for multi-locus analysis, significant differential expression $(p<0.05$; FDR $<0.05)$ of five of these loci loci was evident (Table 3), with significant increases in expression of HERVW copies 3q11.21, 4q31.1, 9q31.3 and 19p12 and a significant decrease in 12q23.3. 


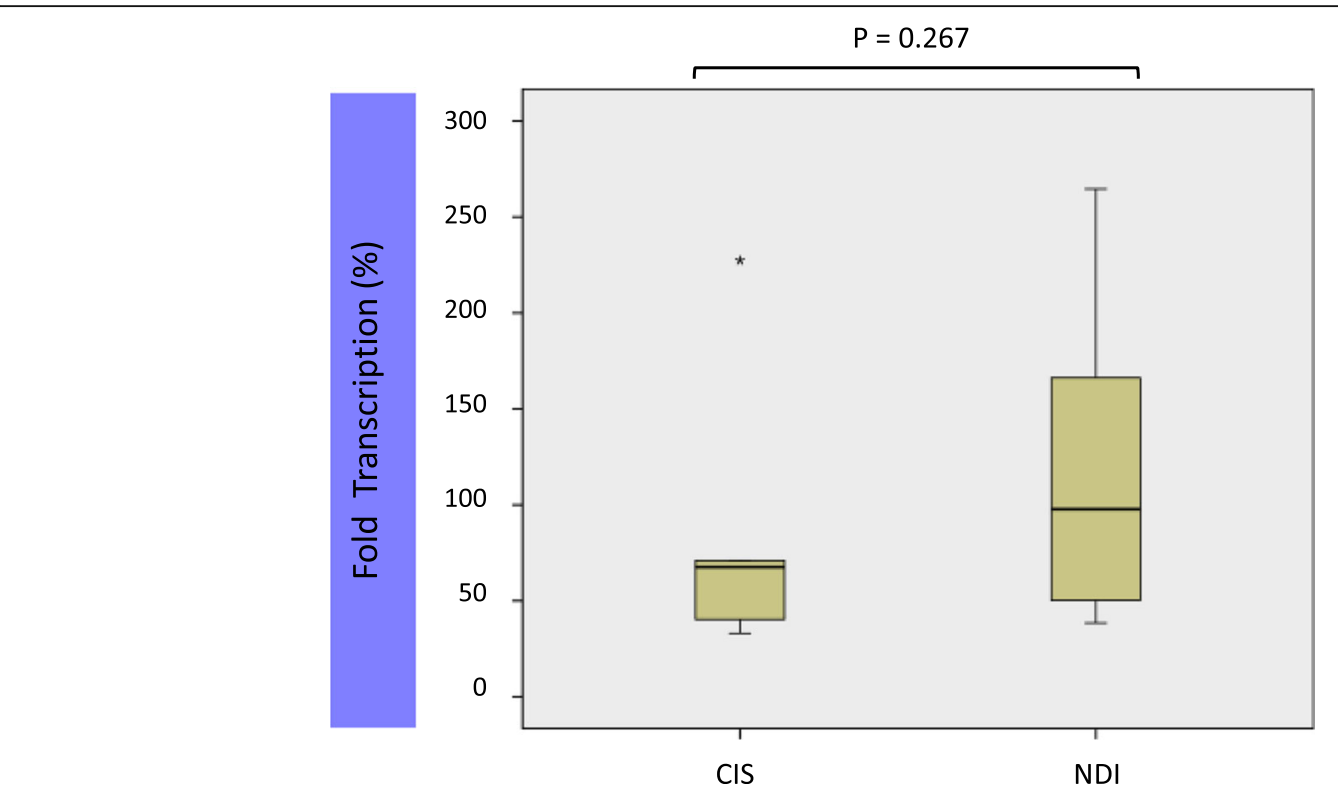

Fig. 1 HERWW expression levels in CIS patients. Expression analysis of HERWW ENV levels in CIS ( $n=6)$ patients and Non-diseased individuals ( $n=15$ ) or NDI (controls) was analyzed by qPCR. Results were normalized using GAPDH as a reference gene, and are represented as the fold expression compared to the median expression level in controls (recalculated as a percentage). U-Mann-Whitney test; $P=0.267$

Table 2 Percentages of reads mapped to individual HERWW loci

\begin{tabular}{|c|c|c|c|c|c|c|c|c|c|c|c|}
\hline & & \multicolumn{10}{|c|}{ reads per locus as a percentage of total mapped reads } \\
\hline & & CIS 58 & CIS 70 & CIS 49 & CIS73 & CIS114 & Control 102 & Control 104 & Control 106 & Control 99 & Control98 \\
\hline & Locus & \multicolumn{10}{|c|}{$\%$ of reads } \\
\hline chr1-1 & chr1:55377330-55377730 & & 0.01 & 0.03 & 0.06 & & & & & & \\
\hline $\operatorname{chr} 2-2$ & chr2:30741934-30742334 & 0.07 & 0.05 & 0.01 & 0.07 & 0.01 & 0.02 & 0.05 & & 0.06 & 0.23 \\
\hline $\operatorname{chr} 2-3$ & chr2:79390513-79390913 & & & & 0.01 & & & & & & \\
\hline chr2-4 & chr2:165514648-165515048 & 0.00 & 0.00 & & 0.02 & & & & & & 0.03 \\
\hline chr2-5 & chr2:176190476-176190876 & 0.64 & 0.38 & 1.55 & 0.22 & & 0.01 & & 0.05 & 1.79 & 0.01 \\
\hline chr3-1 & chr3:96385885-96386285 & 0.27 & 0.49 & 1.26 & 0.16 & 0.01 & 0.03 & 0.04 & 0.08 & & \\
\hline chr3-2 & chr3:141539613-141540013 & 0.01 & 0.00 & & 0.05 & 0.02 & & & & & 0.01 \\
\hline chr3-3 & chr3:142161737-142162137 & 0.01 & 0.00 & & 0.04 & 0.02 & & & & & 0.04 \\
\hline chr3-4 & chr3:149474461-149474861 & 0.68 & 0.60 & 2.31 & 0.34 & 0.15 & 0.34 & 0.26 & 7.25 & 0.19 & 0.05 \\
\hline chr3-5 & chr3:154696338-154696738 & 0.02 & 0.01 & & & & & & & & \\
\hline chr4-2 & chr4:83400034-83400434 & 8.30 & 6.94 & 9.77 & 3.50 & 1.94 & 2.03 & 4.13 & 2.16 & 8.14 & 1.31 \\
\hline chr4-3 & chr4:139543582-139543982 & 0.01 & 0.03 & 0.03 & 0.02 & 0.03 & & & 0.01 & & \\
\hline chr4-5 & chr4:171111550-171111950 & 0.00 & 0.01 & & 0.02 & & & & & & \\
\hline chr5-1 & chr5:44113942-44114342 & 0.79 & 0.83 & 1.66 & 0.24 & 0.04 & 0.26 & 0.14 & 0.19 & 0.08 & 0.15 \\
\hline chr6-2 & chr6:84159363-84159763 & 0.01 & 0.00 & & 0.04 & & & & & & 0.01 \\
\hline chr6-3 & chr6:106682926-106683326 & 0.03 & 0.03 & 0.02 & 0.01 & 0.01 & 0.01 & 0.01 & & & 0.02 \\
\hline chr8-1 & chr8:49148717-49149117 & & & & 0.01 & & & & & & \\
\hline chr8-2 & chr8:74734062-74734462 & 0.47 & 0.50 & 0.19 & 11.25 & 9.15 & 0.32 & 0.25 & 0.26 & 0.50 & 41.29 \\
\hline chr9-1 & chr9:94743580-94743980 & 0.76 & 1.55 & 2.33 & 0.20 & 0.02 & 0.07 & 0.20 & 0.33 & 0.14 & 0.06 \\
\hline chr9-2 & chr9:114099687-114100087 & 0.01 & 0.03 & & 0.03 & & & & & & \\
\hline chr10-1 & chr10:62794101-62794501 & 0.01 & 0.00 & & 0.03 & & & & & & \\
\hline chr10-2 & chr10:96594541-96594941 & 0.00 & 0.01 & & 0.02 & & & & & & \\
\hline chr11-2 & chr11:77569561-77569961 & 0.11 & 0.07 & 0.02 & 0.49 & 0.17 & 0.02 & 0.03 & 0.02 & & 0.67 \\
\hline chr11-3 & chr11:86544743-86545143 & & & & 0.02 & 0.03 & & & & & 0.06 \\
\hline chr12-1 & chr12:38422288-38422688 & 0.01 & & & 0.03 & & & & & & \\
\hline chr12-2 & chr12:38867467-38867867 & 0.01 & 0.00 & & 0.04 & & & & & & \\
\hline chr12-3 & chr12:51296924-51297324 & 1.16 & 1.30 & 1.50 & 0.49 & 0.30 & 0.99 & 0.73 & 0.83 & 0.73 & 0.09 \\
\hline chr12-4 & chr12:105337042-105337442 & 4.07 & 3.72 & 5.58 & 3.91 & 1.17 & 7.43 & 7.45 & 6.96 & 3.84 & 2.77 \\
\hline chr14-2 & chr14:53828947-53829347 & 0.03 & 0.03 & & 0.79 & 0.54 & & 0.01 & & & 0.93 \\
\hline chr15-1 & chr15:55597294-55597694 & 4.55 & 3.96 & 4.74 & 2.59 & 4.74 & 10.26 & 3.90 & 8.54 & 4.46 & 2.19 \\
\hline chr17-1 & chr17:33878854-33879254 & 0.35 & 0.29 & 0.05 & 0.95 & 0.32 & 0.27 & 0.07 & 0.25 & & 0.02 \\
\hline chr17-2 & chr17:53089093-53089493 & 0.01 & 0.00 & & 0.03 & 0.02 & & & & & 0.01 \\
\hline chr19-1 & chr19:22934117-22934517 & 0.03 & 0.06 & 0.03 & 0.05 & & & & & & \\
\hline chr19-2 & chr19:40577781-40578181 & 53.18 & 53.58 & 41.35 & 57.26 & 72.77 & 51.09 & 54.31 & 52.52 & 57.13 & 45.92 \\
\hline chrX-1 & chrX:7617425-7617825 & & 0.01 & & & & & & & & \\
\hline \multirow[t]{2}{*}{$\operatorname{chrX}-2$} & chrX:106295564-106295964 & 23.64 & 23.9371 & 25.24 & 16.81 & 8.52 & 26.76 & 28.21 & 20.20 & 22.67 & 4.06 \\
\hline & № loci/sample & 31 & 32 & 19 & 34 & 21 & 16 & 16 & 15 & 12 & 22 \\
\hline
\end{tabular}

Mapped reads (Table S3), were recalculated as the number of reads mapping to an individual HERVW ENV element relative to the total number of reads, and represented as a percentage. The HERVW loci to which an increased number of reads mapped in CIS patients are indicated in blue 


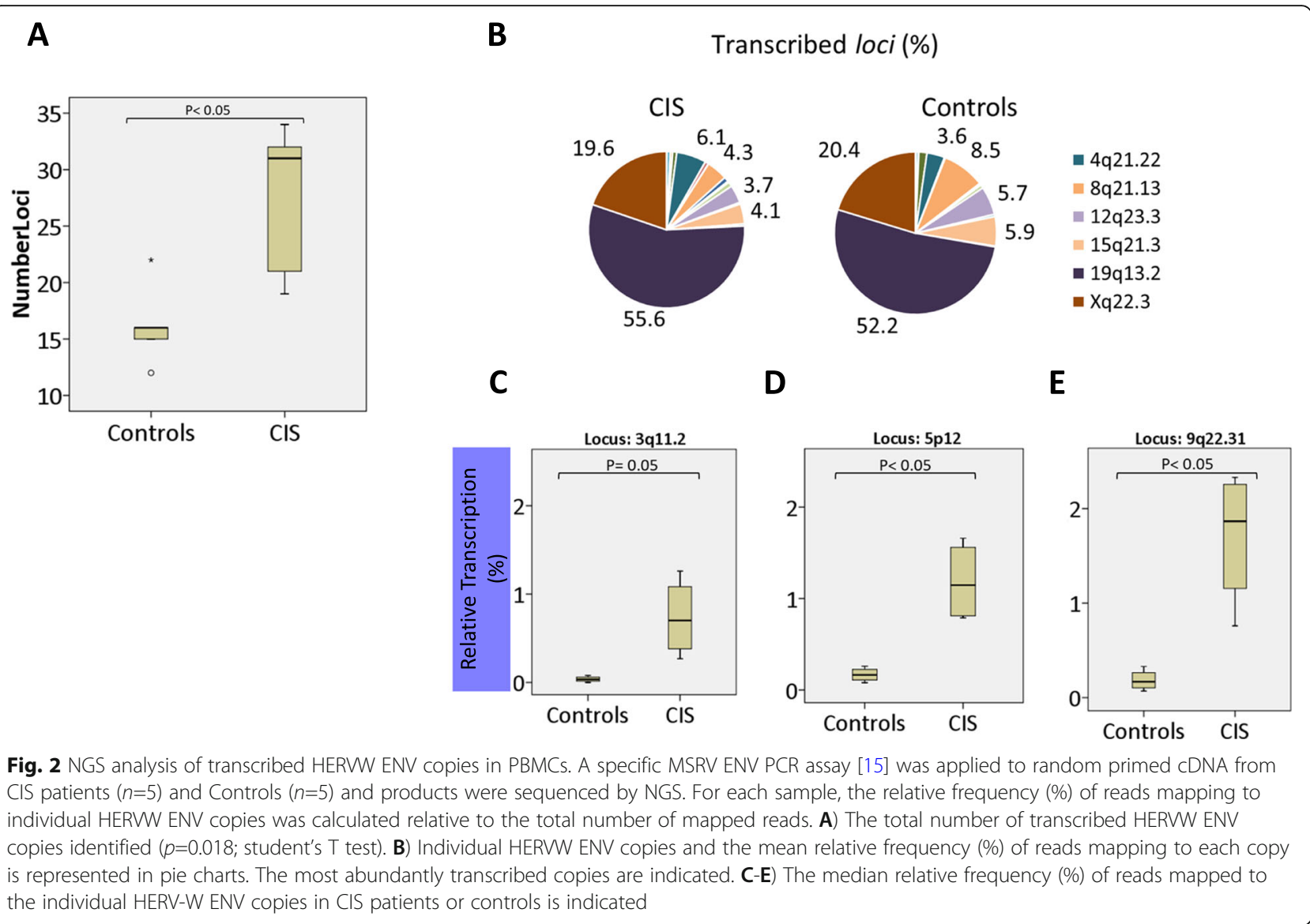

\section{Discussion}

In contrast to the small group of CIS patients analyzed in this study, increased HERVW levels have been associated frequently with MS. Our inability to demonstrate a statistically significant increase of overall HERVW levels in PBMC of CIS patients may be explained by the selection of this particular group or more likely simply by small sample size. However, lack of increased expression is not unprecedented as it was previously reported in a cohort of
South African MS patients, although different primers were used for this analysis [17].

We perfomed NGS analysis to identify individual HERVW copies that show altered expression in PBMC, comparing CIS patients $(n=5)$ to controls $(n=5)$. Although more definite answers require future analysis of more subjects, in the CIS patients analyzed more HERVW loci are expressed than in control subjects. A similar increase has been reported previously

Table 3 HERWW copies differentially expressed in CIS patients

\begin{tabular}{|c|c|c|c|c|c|}
\hline HERVW Copy & Repeatmasker name & Reads in CIS & Reads in Controls & Fold increase & $P$ value \\
\hline chr 3-1 & $3 q 11.2$ & 2221 & 124 & 14,76 & 0,0040 \\
\hline chr 4-3 & $4 q 31.1$ & 92 & 6 & 12,64 & 0,0333 \\
\hline chr 5-1 & $5 p 12$ & 3999 & 562 & 5,86 & 0,27 \\
\hline chr 9-1 & $9 q 22.31$ & 5678 & 614 & 9,2 & 0,12 \\
\hline chr 9-2 & $9 q 31.3$ & 81 & 1 & $>20$ & 0,0333 \\
\hline chr12-4 & $12 q 23.3$ & 18962 & 20812 & $-1,33$ & 0,0015 \\
\hline chr19-1 & $19 p 12$ & 192 & 1 & $>20$ & 0,0015 \\
\hline
\end{tabular}

The table lists several HERVW copies, using references from Tables S2-S3. Names used in RepeatMasker are also indicated. The sum of mapped reads in samples from five CIS patients or five controls is listed, as well as the resulting fold change in CIS versus control. The $P$ value for the difference between NDI and CIS samples was adjusted for multiple testing (FDR <0.05) 
in MS brain [18]. While previous studies failed to identify MS-specific loci or expression [18, 19], in the CIS patients we found statistically significant overrepresentation of reads corresponding to specific loci (i.e. 3q11.2 and 19p12, see Table 3 for complete list). Locus-specific qPCR assays may first help confirm this finding in a larger patient cohort, and subsequently be evaluated as a potential prognostic assay.

These combined overrepresented loci produce only $1-3 \%$ of total transcripts (Fig. 2 C-E). The combined findings on low levels of overexpression, activation of more loci, and activation of low-expressing HERV $\mathrm{W}$ elements in CIS patients suggest that their potential contribution to the pathology may be unrelated to overall high expression levels. None of the copies identified encode full-length ENV protein, as the sequences corresponding to the ENV gene are truncated, lack ATG codons, and/or carry frame shifts and STOP codons (Suppl Figure 2). CIS-associated copies may produce proteins (either or not ENVrelated) that are especially active in activation of TLR4 [20], or RNAs that trigger the native immune system through TLR3 [21, 22]. Although our analysis shows that upregulation of specific HERVW loci in PBMC is associated with CIS, the presence of these transcripts in MS brain is unknown at present. A potential role of these transcripts in proviral protein production and activation of either the peripheral immune system or CNS disease remains to be established.

\section{Supplementary Information}

The online version contains supplementary material available at https://doi. org/10.1186/s13100-021-00243-1

Additional file 1: Table S1. The levels of GAPDH, RPL19 and HSDA were determined by qPCR in the samples indicated, as described in the legend to Suppl Fig. 1. The Table lists the Cts obtained for each gene (columns named accordingly, the mean of all three (column "mean") and the difference between the Cts obtained using either GAPDH (G) or the mean of three reference genes (P) (Column P-G). The difference (mean 2.76; standard deviation 0.29 ) is statistically constant among samples (Welch's t-test; $p<0.05$ ).

Additional file 2: Table S2. List of HERWW ENV loci according to the GRCh37.p5 version of the human genome database. Loci identified by unbiased read mapping were verified as HERWW loci by comparison with the Repbase Update library of repeats from the Genetic Information Research Institute (GIRI)[23], using the the RepeatMasker program. Nomenclature used in Tables is indicated, the genomic location of each copy as well as alternative names used in the literature. Sequences corresponding to the coordinates listed were downloaded and compared to primer sequences using the "align" function in SerialCloner (version 2-6-1). Identity with primer sequence is indicated in black, mismatches in red.

Additional file 3: Table S3. Number of reads mapping to the HERWW copies indicated in each of the five CIS or NDI (control) samples. The total number of reads per sample is indicated. The sum of reads in the CIS and NDI groups is indicated.

Additional file 4: Table S4. Primers used.

Additional file 5: Figure S1. Comparison of reference genes.
Additional file 6: Figure S2. Comparison of HERWW elements.

Additional file 7: Figure S3. $q P C R$ primer efficiency standard curve analysis.

Additional file 8: Supplementary Methods.

Additional file 9. MIQE checklist.

\section{Acknowledgements}

We acknowledge the use of the Sequencing and Functional Genomics Core Facility (Servicio Científico Técnico de Secuenciación y Genómica Funcional) of the Instituto Aragonés de Ciencias de la Salud Aragon Health Sciences Institute (IACS) and Universidad de Zaragoza. We are particularly grateful to all of the patients for their participation in and commitment to the study. Special thanks go to Carmen Ocabo for her help with informing patients, the collection and the control of clinical samples.

\section{Authors' contributions}

JRA and JM were responsible for the conception and recruitment of the patient samples. JS and SMR designed the study, experiments were carried out by SMR and MS. JS, MS, AC and SMR performed the data analysis. JS and SMR interpreted the results and wrote the manuscript. All the authors critically revised the manuscript for intellectual and scientific content and approved the final manuscript.

\section{Funding}

The work described in this manuscript has been financed by the Instituto Carlos III (Government of Spain)(FIS PI13/02518), and by several grants from the Government of Aragon, cofinanced by FEDER ("Una manera de hacer Europa, Construyendo Europa desde Aragón"/European Social Funds): B77 and B05_17D. SMR has been supported by PhD fellowship C071/2014 from the DGA (Aragón, Spain). The funding bodies played no role in the design of the study, in the collection, analysis, and interpretation of data, in writing the manuscript and in the decision to submit the article for publication.

\section{Availability of data and materials}

The datasets used and/or analyzed in this study have been deposited in NCBI's Gene Expression Omnibus (Edgar et al., 2002) and are accessible through GEO Series accession number GSE173929 (https://www.ncbi.nlm.nih. gov/geo/query/acc.cgi?acc=GSE173929)

\section{Declarations}

Ethics approval and consent to participate

The local ethics committee (CEICA) approved the study protocol (CP - CI PI14/ 0021 dated 26/02/2014; modified on 25/10/2017), and patients provided written informed consent (protocolo y información para el paciente v2 de 29/ 12/2013).

Consent for publication

Not applicable

\section{Competing interests}

The authors report no conflict of interest

\section{Author details}

${ }^{1}$ Instituto Aragonés de Ciencias de la Salud (IACS), c/Juan Bosco 13, 50009 Zaragoza, Spain. ${ }^{2}$ Sequencing and Functional Genomics, Aragon Biomedical Research Center (CIBA), Instituto Aragonés de Ciencias de la Salud (IACS), Zaragoza, Spain. ${ }^{3}$ Aragon Biomedical Research Center (CIBA), Instituto Aragonés de Ciencias de la Salud (IACS), Unidad de Biocomputación, Zaragoza, Spain. ${ }^{4}$ Instituto de Investigación Sanitaria de Aragón (IIS Aragón), Zaragoza, Spain. ${ }^{5}$ Department of Neurology, University Hospital Miguel Servet, Zaragoza, Spain. ${ }^{6}$ ARAID Foundation, Avda. de Ranillas 1-D, 50018 Zaragoza, Spain. ${ }^{7}$ Placental pathophysiology and fetal programming research group del IISA, c/Juan Bosco 13, 50009 Zaragoza, Spain. 
Received: 23 October 2020 Accepted: 1 June 2021

Published online: 29 June 2021

\section{References}

1. Grandi N, Tramontano E. Human endogenous retroviruses are ancient acquired elements still shaping innate immune responses. Front Immunol. 2018;9:2039

2. Bourque G, Burns KH, Gehring M, Gorbunova V, Seluanov A, Hammell M, et al. Ten things you should know about transposable elements. Genome Biol. 2018;19:1-12.

3. Sedivy JM, Kreiling JA, Neretti N, Cecco M, De, Criscione SW, Hofmann JW, et al. Death by transposition - the enemy within? BioEssays. 2013; 35:1035-43.

4. Cardelli M, Marchegiani F. Good, bad, mobile elements: genome's most successful "parasites" as emerging players in cell and organismal aging. Curr Pharm Des. 2013;19:1739-52.

5. Perron H, Garson JA, Bedin F, Beseme F, Paranhos-Baccala G, KomurianPradel $F$, et al. Molecular identification of a novel retrovirus repeatedly isolated from patients with multiple sclerosis. The Collaborative Research Group on Multiple Sclerosis. Proc Natl Acad Sci U S A. 1997:94:7583-8.

6. Küry $P$, Nath A, Créange A, Dolei A, Marche P, Gold J, et al. Human Endogenous Retroviruses in Neurological Diseases. Trends Mol. Med. 2018. p. 379-94

7. Grandi N, Cadeddu M, Blomberg J, Tramontano E. Contribution of type W human endogenous retroviruses to the human genome: characterization of HERV-W proviral insertions and processed pseudogenes. Retrovirology. 2016; 13:67.

8. Perron H, Germi R, Bernard C, Garcia-Montojo M, Deluen C, Farinelli L, et al. Human endogenous retrovirus type $\mathrm{W}$ envelope expression in blood and brain cells provides new insights into multiple sclerosis disease. Mult Scler J. 2012;18:1721-36.

9. García-Montojo M, de la Hera B, Varadé J, de la Encarnación A, Camacho I, Domínquez-Mozo M, et al. HERV-W polymorphism in chromosome $X$ is associated with multiple sclerosis risk and with differential expression of MSRV. Retrovirology. 2014;11:2.

10. Morandi E, Tanasescu R, Tarlinton RE, Constantinescu CS, Zhang W, Tench $C$, et al. The association between human endogenous retroviruses and multiple sclerosis: A systematic review and metaanalysis. PLoS One. 2017;12.

11. Kuhle J, Disanto G, Dobson R, Adiutori R, Bianchi L, Topping J, et al. Conversion from clinically isolated syndrome to multiple sclerosis: A large multicentre study. Mult Scler. 2015;21:1013-24.

12. Böyum A. Isolation of mononuclear cells and granulocytes from human blood. Isolation of monuclear cells by one centrifugation, and of granulocytes by combining centrifugation and sedimentation at $1 \mathrm{~g}$. Scand J Clin Lab Invest Suppl. 1968:97:77-89.

13. Macías-Redondo S, Strunk M, Guillén M, Ara J, Martín J, Alvaréz-Lafuente R, et al. Searching for non-referenced HERWW copies relevant to MS: a splinkerette-PCR method. Submitted. 2020.

14. Pérez-Palacios R, Macías-Redondo S, Climent M, Contreras-Moreira B, Muniesa P, Schoorlemmer J. In Vivo Chromatin Targets of the Transcription Factor Yin Yang 2 in Trophoblast Stem Cells. PLoS One. 2016;11:e0154268.

15. Mameli G, Poddighe L, Astone V, Delogu G, Arru G, Sotgiu S, et al. Novel reliable real-time PCR for differential detection of MSRVenv and syncytin-1 in RNA and DNA from patients with multiple sclerosis. J Virol Methods. 2009;161:98-106

16. Love Ml, Huber W, Anders S. Moderated estimation of fold change and dispersion for RNA-seq data with DESeq2. Genome Biol. 2014;15:550.

17. De Villiers JNP, Treurnicht FK, Warnich L, Carr J, Van Rensburg SJ, Kotze MJ. Analysis of viral and genetic factors in South African patients with multiple sclerosis. Metab Brain Dis. 2006;21:163-9.

18. Schmitt K, Richter C, Backes C, Meese E, Ruprecht K, Mayer J. Comprehensive Analysis of Human Endogenous Retrovirus Group HERV-W Locus Transcription in Multiple Sclerosis Brain Lesions by High-Throughput Amplicon Sequencing. J Virol. 2013;87:13837-52.

19. Laufer G, Mayer J, Mueller BF, Mueller-Lantzsch N, Ruprecht K. Analysis of transcribed human endogenous retrovirus $W$ env loci clarifies the origin of multiple sclerosis-associated retrovirus env sequences. Retrovirology. 2009;6:37.

20. Rolland A, Jouvin-Marche $E$, Viret $C$, Faure $M$, Perron $H$, Marche PN. The envelope protein of a human endogenous retrovirus-W family activates innate immunity through CD14/TLR4 and promotes Th1-like responses. I Immunol. 2006:176:7636-44.

21. Hurst TP, Magiorkinis G. Activation of the innate immune response by endogenous retroviruses. J Gen Virol. 2015:96:1207-18.

22. Stetson DB. Endogenous retroelements and autoimmune disease. Curr Opin Immunol. 2012;24:692-7.

\section{Publisher's Note}

Springer Nature remains neutral with regard to jurisdictional claims in published maps and institutional affiliations.
Ready to submit your research? Choose BMC and benefit from:

- fast, convenient online submission

- thorough peer review by experienced researchers in your field

- rapid publication on acceptance

- support for research data, including large and complex data types

- gold Open Access which fosters wider collaboration and increased citations

- maximum visibility for your research: over $100 \mathrm{M}$ website views per year

At $\mathrm{BMC}$, research is always in progress.

Learn more biomedcentral.com/submissions 